\title{
BMJ Open Treatment outcomes of initial differential antiretroviral regimens among HIV patients in Southwest China: comparison from an observational cohort study
}

\author{
Ruihua Kang, ${ }^{1}$ Liuhong Luo, ${ }^{2}$ Huanhuan Chen, ${ }^{2}$ Qiuying Zhu, ${ }^{2}$ Lingjie Liao, ${ }^{1}$ \\ Hui Xing, ${ }^{1}$ Jinhui Zhu, ${ }^{2}$ Zhiyong Shen, ${ }^{2}$ Guanghua Lan, ${ }^{2}$ Zhenzhu Tang, ${ }^{2}$ \\ Yiming Shao, ${ }^{1}$ Wenmin Yang, ${ }^{2}$ Yuhua Ruan ${ }^{1}$
}

To cite: Kang R, Luo L, Chen $\mathrm{H}$, et al. Treatment outcomes of initial differential antiretroviral regimens among HIV patients in Southwest China: comparison from an observational cohort study. BMJ Open 2019;9:e025666. doi:10.1136/ bmjopen-2018-025666

- Prepublication history and additional material for this paper are available online. To view these files, please visit the journal online (http://dx.doi. org/10.1136/bmjopen-2018025666).

WY and YR contributed equally.

Received 28 July 2018

Revised 21 February 2019

Accepted 25 February 2019

Check for updates

(C) Author(s) (or their employer(s)) 2019. Re-use permitted under CC BY-NC. No commercial re-use. See rights and permissions. Published by BMJ.

For numbered affiliations see end of article.

Correspondence to Mr Wenmin Yang; ywm5839@163.com and Dr Yuhua Ruan; ruanyuhua92@163.com

\section{ABSTRACT}

Objectives China has continued to expand antiretroviral therapy (ART) services and optimise ART guidelines in an effort to significantly reduce and prevent mortality and transmission rates among HIV patients. However, no study to date has compared treatment outcomes of initial differential antiretroviral regimens among HIV patients in a real-world setting in China. This study aimed to compare the effects of different ART regimens on treatment outcomes among adults.

Design Observational retrospective cohort study. Setting Data from 2011 to 2013 in Guangxi, China. Participants Patients aged $\geq 18$ years $(n=25732)$ were selected.

Results A total of 25732 patients were included in this study. The average mortality and attrition rate were 2.64 and 4.98, respectively, per 100 person-years. Using Cox proportional hazard models, zidovudine-based (AZT-based) regimen versus stavudine-based (D4T-based) regimen had an adjusted HR (AHR) for death of 0.65 (95\% Cl 0.58 to 0.73 ); the AHR of tenofovir-based (TDF-based) versus D4T-based regimens was 0.81 (95\% Cl 0.71 to 0.92$)$, and of lopinavir-ritonavir-based (LPV/r-based) versus D4Tbased regimens, 1.19 (95\% $\mathrm{Cl} 1.04$ to 1.37). AZT-based versus D4T-based regimens had an AHR for dropout of 0.89 ( $95 \% \mathrm{Cl} 0.81$ to 0.97 ); this ratio for TDF-based versus D4T-based regimens was 0.88 (95\% Cl 0.80 to 0.98$)$, and for LPV/r-based versus D4T-based regimens, $1.42(95 \% \mathrm{Cl}$ 1.27 to 1.58). AZT-based and TDF-based regimens had a lower risk compared with D4T-based regimens, while LPV/ r-based regimens had a higher risk. High gastrointestinal reactions and poor adherence were observed among HIV patients whose initial ART regimen was LPV/r-based. Conclusions Our study found that the treatment outcomes of initial ART regimens that were AZT-based or TDF-based were significantly better than D4T-based or $\mathrm{LPV} / \mathrm{r}$-based regimens. This finding could be related to the higher rates of gastrointestinal reactions and poorer adherence associated with the LPV/r-based regimens compared with other initial ART regimens.

\section{INTRODUCTION}

Highly active antiretroviral therapy (ART) has been an available treatment for people living
Strengths and limitations of this study

- Our study was conducted as an observational retrospective cohort study in Guangxi, China, using the data of 25732 patients.

- The large sample size provides the strong evidence in support of our study results.

- The study population included only subjects who initiated antiretroviral therapy (ART); but subjects who were infected with HIV but not receiving ART were not included.

- This study might not be representative of all patients living with HIV in China.

with HIV for more than three decades. In China, ART regimens are applied according to WHO guidelines. As the WHO guidelines change, ART criteria and regimens have been appropriately adjusted. The National Free Antiretroviral Treatment Program (NFATP) was initiated in China among former plasma donors as a pilot project in Henan province in 2002, and fully performed nationally in 2003. ${ }^{2}$ In 2005, the recommended first-line regimen in China was zidovudine (AZT) or stavudine (D4T) with lamivudine (3TC) and nevirapine (NVP), ${ }^{2}$ as recommended by the WHO. In the beginning of 2008, the Chinese national criteria for receiving ART treatment were revised as follows: CD4 cell count $<350 / \mathrm{mm}^{3}$, WHO-defined stage III/IV clinical conditions or willingness to receive ART regardless of meeting the first two criteria. ${ }^{4}$ The regimen was adjusted again and D4T was gradually replaced by AZT or tenofovir (TDF). ${ }^{4}$ To date, all individuals infected with HIV who are eligible for treatment have been treated in all 31 provinces, autonomous regions and municipalities in China. ${ }^{5}$ Current first-line ART regimens include TDF or AZT with 3TC and efavirenz (EFV) or NVP. 
Second-line ART regimens include lopinavir-ritonavir $(\mathrm{LPV} / \mathrm{r})$ with $3 \mathrm{TC}$ and AZT or TDF. ${ }^{5}$ To achieve the Joint United Nations Programme on HIV/AIDS (UNAIDS) '90-90-90' target $^{6}{ }^{6}$ regimens that include $\mathrm{LPV} / \mathrm{r}$ have been gradually and widely implemented as initial ART treatment in China. However, despite recommendations to initiate ART among all individuals infected with HIV, there exists limited understanding about the effects of different initial regimens on the mortality and attrition rates in real-world settings in China.

Guangxi Zhuang Autonomous Region (Guangxi) is located in Southwest China, and borders the drug trafficking route known as the 'Golden Triangle'. By the end of 2017, Guangxi was ranked fourth among all provinces in China for number of newly reported HIV cases, and patients with HIV/AIDS in Guangxi accounted for $10 \%$ of the entire HIV/AIDS population in China. ${ }^{7}$ Thus Guangxi plays a critical role in the country's HIV prevention and treatment campaign.

The objective of this study was to estimate the treatment effects of different initial ART regimens (including D4T-based regimen [D4T+3TC+EFV/NVP], AZT-based regimen $[\mathrm{AZT}+3 \mathrm{TC}+\mathrm{EFV} / \mathrm{NVP}]$, TDF-based regimen $[\mathrm{TDF}+3 \mathrm{TC}+\mathrm{EFV} / \mathrm{NVP}]$ and $\mathrm{LPV} / \mathrm{r}$-based regimen $[\mathrm{LPV} /$ $\mathrm{r}+3 \mathrm{TC}+\mathrm{D} 4 \mathrm{~T} / \mathrm{AZT} / \mathrm{TDF}]$ ) on death, dropout, death and dropout, and viral load among HIV patients, using the database of a large ART treatment cohort.

\section{MATERIALS AND METHODS}

\section{Patient and public involvement}

The study being retrospective, patients or the public were not involved in the design or in the conduct of the study.

\section{Study design and study participants}

This retrospective observational cohort study of HIV antiretroviral treatment was conducted in Guangxi, an autonomous region in rural Southwest China. The study participants included HIV patients who initiated free ART between 2011 and 2013 through the Chinese NFATP. The date censored was 30 April 2016. Eligibility criteria of individuals included those who initiated free ART between 2011 and 2013 through the NFATP in Guangxi, those who were at least 18 years of age at the time of ART initiation, those who provided informed consent to participate in this study, and those whose initial ART regimen was D4T-based, AZT-based, TDF-based or LPV/r-based. All research methods in this study were carried out in accordance with the approved guidelines.

\section{Data collection}

The baseline variables of all patients included age, sex, marital status, route of HIV infection, laboratory results of CD4 cell counts before ART, WHO clinical stage before ART, initial ART regimen, current ART regimen, date of ART initiation, date of discontinuing ART and reasons for treatment discontinuation. Follow-up status variables included: treatment continuation, loss to follow-up, survival status, transferals to another clinic and stopped ART. The follow-up visits occurred at 0.5, 1, 2 and 3 months following ART initiation, and then every 3 months thereafter. ${ }^{8}$ Loss to follow-up was defined as not having a visit for more than 90 days after the last date seen in clinic.

\section{Statistical analysis}

In this study, treatment outcomes included death and dropout from follow-up. Dropout included stopped ART or loss to follow-up as reported through the database. Time zero was defined as the date of ART initiation, and data were censored at 30 April 2016. Survival time was calculated from the date of ART initiation to date of death or the last follow-up. Person-years were the unit of measure for incidence rates mortality rates, dropout rates and death and dropout rates with their $95 \%$ CI were analysed with incidence density rate per 100 person-years of follow-up. We used Cox proportional hazard models to estimate HR to compare the effects of initial ART regimens on death, dropout, and death and dropout.

We also collected data regarding viral load (VL) at 12 months of ART, adverse events, gastrointestinal reactions and adherence during the first 3 months. Self-reported adherence variables included missed doses in the past 7 days during the first 3 months. We used multivariate logistic regression models to estimate the differences of $\mathrm{VL}<50$ copies/mL at 12 months of ART, adverse events during the first 3 months, gastrointestinal reactions during the first 3 months, and adherence among different initial ART regimens.

In the adjusted model, the following baseline covariates were adjusted to control for potential confounding factors: age, sex, marital status, route of HIV infection and WHO clinical stage before ART. Statistical significance was determined using a two-tailed $\mathrm{p}$ value $<0.05$. All statistical analyses were performed using SAS V.9.1 for Windows.

\section{RESULTS}

\section{General characteristics of the study population}

A total of $25862 \mathrm{HIV} /$ AIDS patients initiated ART between 2011 and 2013 in Guangxi, China. One hundred and thirty patients were excluded: 46 of them were less than 18 years of age, 5 were visited more than 12 months after the first visit and 79 had an initial ART regimen that was not D4T-based, AZT-based, TDF-based or LPV/r-based (online supplementary figure 1). A final total of 25732 patients were included in this study. The baseline characteristics of these 25732 patients are provided in table 1 . Patient ages were categorised into $18-29$ years, $30-49$ years and $\geq 50$ years; these age groups accounted for $13.7 \%, 47.4 \%$ and $39.0 \%$, respectively, of the study population. The majority of patients $(\mathrm{n}=17139 ; 66.6 \%)$ were male, and 18074 patients $(70.2 \%)$ were married. The main route of HIV infection was heterosexual intercourse $(88.9 \%)$, followed by injection drug use $(7.5 \%)$, homosexual intercourse 
Table 1 Characteristics of HIV patients who initiated ART between 2011 and 2013 in Guangxi, China

\begin{tabular}{|c|c|c|}
\hline Variable & No & $\%$ \\
\hline Total & 25732 & 100.0 \\
\hline \multicolumn{3}{|l|}{ Age (years) } \\
\hline 18-29 & 3513 & 13.7 \\
\hline $30-49$ & 12186 & 47.4 \\
\hline$\geq 50$ & 10033 & 39.0 \\
\hline \multicolumn{3}{|l|}{ Sex } \\
\hline Male & 17139 & 66.6 \\
\hline Female & 8593 & 33.4 \\
\hline \multicolumn{3}{|c|}{ Marital status } \\
\hline Married & 18074 & 70.2 \\
\hline Other & 7658 & 29.8 \\
\hline
\end{tabular}

Route of HIV infection

\begin{tabular}{|c|c|c|}
\hline Heterosexual intercourse & 22882 & 88.9 \\
\hline Homosexual intercourse & 321 & 1.3 \\
\hline Intravenous drug use & 1931 & 7.5 \\
\hline Other & 598 & 2.3 \\
\hline \multicolumn{3}{|c|}{ CD4 count before ART (cells $/ \mathrm{mm}^{3}$ ) } \\
\hline$<350$ & 22458 & 87.3 \\
\hline$\geq 350$ & 2756 & 10.7 \\
\hline Missing & 518 & 2.0 \\
\hline \multicolumn{3}{|l|}{ WHO clinic stage before ART } \\
\hline $\mathrm{I} / \mathrm{II}$ & 14985 & 58.2 \\
\hline III/IV & 10747 & 41.8 \\
\hline \multicolumn{3}{|l|}{ Initial ART regimen } \\
\hline D4T-based regimen & 5483 & 21.3 \\
\hline AZT-based regimen & 12018 & 46.7 \\
\hline TDF-based regimen & 5352 & 20.8 \\
\hline LPV/r-based regimen & 2879 & 11.2 \\
\hline \multicolumn{3}{|l|}{ Current ART regimen } \\
\hline The first-line ART & 20194 & 78.5 \\
\hline The second-line ART & 5538 & 21.5 \\
\hline \multicolumn{3}{|l|}{ Year of ART initiation } \\
\hline 2011 & 7722 & 30.0 \\
\hline 2012 & 9178 & 35.7 \\
\hline 2013 & 8832 & 34.3 \\
\hline
\end{tabular}

ART, antiretroviral therapy; AZT, zidovudine; D4T, stavudine; LPV/r, lopinavir-ritonavir; TDF, tenofovir.

$(1.3 \%)$, and other routes of transmission $(2.3 \%)$. The number of patients with CD4 counts before ART $<350$ cells $/ \mathrm{mm}^{3}$ and $\geq 350$ cells $/ \mathrm{mm}^{3}$ were $22458(87.3 \%)$ and 2756 (10.7\%), respectively. An additional 518 (2.0\%) patients had unknown CD4 counts before ART initiation. Patients who were WHO-defined clinical stage III/IV before ART accounted for $41.8 \%$ of the study population. Patients with initial ART regimens of D4T-based, AZT-based, TDF-based and LPV/r-based accounted for $21.3 \%, 47.2 \%, 21.0$ and $11.2 \%$, respectively. The number of patients being treated with the current first-line ART regimen was 20194 (78.5\%). The proportion of patients who initiated ART in 2011, 2012 and 2013 was $30.0 \%, 35.7 \%$ and $34.3 \%$, respectively.

\section{Mortality rates}

Among 25732 patients who initiated ART between 2011 and 2013 in Guangxi, 2062 deaths were observed. In the first, second, third, fourth and fifth year of ART initiation, $1164,427,273,153$ and 45 patients died, respectively. The mortality rates and $95 \% \mathrm{CI}$ for these years were $4.90(95 \%$ CI 4.62 to 5.18$), 1.97$ (95\% CI 1.78 to 2.15$), 1.50$ (95\% CI 1.32 to 1.68 ), 1.43 (95\% CI 1.20 to 1.66 ) and 1.21 (95\% CI 0.86 to 1.57 ) per 100 person-years, respectively. The average mortality rate was 2.64 deaths per 100 personyears among all patients (95\% CI 2.53 to 2.75) (online supplementary table 1).

\section{Dropout rates}

Among 25732 patients, 3893 dropped out from follow-up. Of these, 2531 patients were lost to follow-up, and 1362 patients stopped ART. The number of patients who dropped out in the first, second, third, fourth and fifth year of ART initiation was 3105, 1136, 727, 342 and 124, respectively. In these years, the dropout rates and $95 \% \mathrm{CI}$ were 13.08 (95\% CI 12.62 to 13.54), 5.23 (95\% CI 4.91 to 5.52 ), 4.00 (95\% CI 3.71-4.29), 3.20 (95\% CI 2.86 to 3.54 ) and 3.34 (95\% CI 2.75 to 3.93 ) per 100 personyears, respectively. The average dropout rate in the study period was 4.98 per 100 person-years among all patients (95\% CI was 4.83-5.15) (online supplementary table 2).

\section{Death and dropout rates}

Among 25732 patients, 5955 deaths and dropouts were observed. A total of 4269, 1563, 1000, 495 and 169 patients died or dropped out in the first, second, third, fourth and fifth year of ART initiation, respectively. The average death and dropout rate was 7.62 per 100 personyears among all patients (95\% CI 7.43 to 7.81 ) (online supplementary table 3 ).

\section{Effects of initial ART regimen on death}

The deaths per 100 person-years for D4T-based, AZT-based, TDF-based and LPV/r-based initial ART regimens was 3.77 (95\% CI 3.48 to 4.06), 1.80 (95\% CI 1.66 to 1.93 ), 2.71 (95\% CI 2.44 to 2.98 ) and 4.18 (95\% CI 3.72 to 4.63 ), respectively (table 2 ). After adjustment with Cox proportional hazards models, the AHR for death of patients on AZT-based versus D4T-based regimens was 0.65 (95\% CI 0.58 to 0.73), the AHR for TDF-based versus D4T-based regimens was 0.81 (95\% CI 0.71 to 0.92$)$ and the AHR for LPV/r-based versus D4T-based regimens was 1.19 (95\% CI 1.04 to 1.37). After adjustment, the AHR for death of LPV/r-based versus AZT-based initial ART regimen was 1.83 (95\% CI 1.60 to 2.10 ). 
The dropout per 100 person-years for D4T-based, AZT-based, TDF-based and LPV/r-based initial ART regimen was 5.03 (95\% CI 4.70 to 5.37$), 4.37$ (95\% CI 4.16 to 4.57 ), 5.04 (95\% CI 4.67 to 5.40 ) and 7.85 (95\% CI 7.23 to 8.48 ), respectively (table 3 ). The AHR for dropout of initial ART regimen that was AZT-based, TDF-based and $\mathrm{LPV} / \mathrm{r}$-based versus D4T-based was 0.89 (95\% CI 0.81 to 0.97$), 0.88$ (95\% CI 0.80 to 0.98 ) and $1.42(95 \%$ CI 1.27 to 1.58), respectively. After adjustment, the AHR for dropout of initial ART regimen that was LPV/r-based versus AZT-based was 1.60 (95\% CI 1.45 to 1.76 ).

\section{Effects of initial ART regimen on death and dropout}

After adjustment, the AHR for death and dropout of AZT-based, TDF-based and LPV/r-based versus D4T-based initial ART regimen was 0.79 (95\% CI 0.74 to 0.85$), 0.85$ (95\% CI 0.78 to 0.92 ) and 1.32 (95\% CI 1.22 to 1.44 ), respectively. After adjustment, the AHR for death and dropout of $\mathrm{LPV} / \mathrm{r}$-based versus ART-based initial ART regimen was 1.67 (95\% CI 1.54 to 1.81 ) (online supplementary table 4$)$.

\section{Viral load at 12 months of ART}

During 12 months of ART, 1164 patients died and 3105 patients dropped out, with a remaining total of 21463 patients. The proportion of patients with VL $<50$ copies/ $\mathrm{mL}$ was $75.0 \%$ (table 4 ). The number of patients whose initial ART regimen was LPV/r-based, D4T-based, AZT-based and TDF-based was 2220, 4393, 10293 and 4601 , respectively, and the respective proportion of $\mathrm{VL}<50$ copies/mL in these groups was $73.7 \%, 72.5 \%$, $75.3 \%$ and $77.6 \%$. After adjusting for factors of age, sex, marital status, route of HIV infection, CD4 count before ART and WHO clinical stage before ART, differences in VL $<50$ copies $/ \mathrm{mL}$ were not statistically significant between LPV/r-based and D4T-based regimens $(\mathrm{p}=0.32)$ or between $\mathrm{LPV} / \mathrm{r}$-based and AZT-based regimens ( $p=0.33$ ), but were statistically significant between $\mathrm{LPV} / \mathrm{r}$ based and TDF-based regimens $(\mathrm{p}<0.001)$.

\section{Adverse events and adherence}

Information for adverse events during the first 3 months was available for 24517 patients (table 5). A total of 6966 (28.4\%) patients had adverse events, and the proportion of patients that had adverse events among those whose initial ART regimen was LPV/r-based, D4T-based, AZT-based and TDF-based was $27.6 \%, 27.3 \%, 31.6 \%$ and $22.7 \%$, respectively. After adjusting for factors of age, sex, marital status, route of HIV infection, CD4 count before ART and WHO clinical stage before ART, differences in adverse events were not statistically significant between LPV/r-based and D4T-based regimens $(\mathrm{p}=0.26)$, but were statistically significant between $\mathrm{LPV} / \mathrm{r}$-based and AZT-based regimens $(p<0.001)$ and between $L P V / r$-based and TDF-based regimens $(\mathrm{p}<0.001)$.

A total of $4203(17.1 \%)$ patients had gastrointestinal reactions (table 5). Among those who initiated an ART 


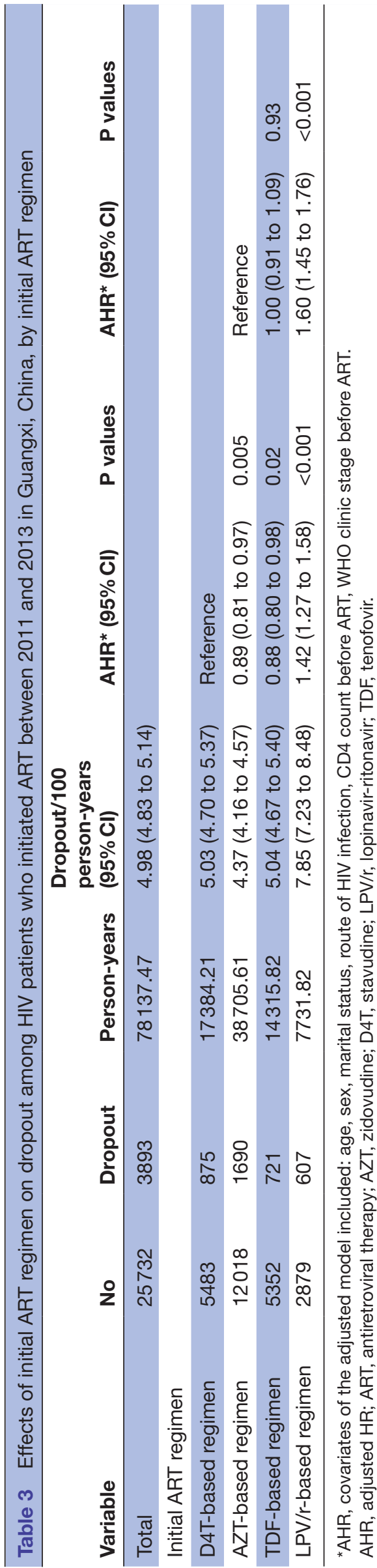

regimen that was LPV/r-based, D4T-based, AZT-based or TDF-based, the percentage of patients with gastrointestinal reactions was $22.9 \%, 15.1 \%, 19.3 \%$ and $11.4 \%$, respectively. After adjusting for factors of age, sex, marital status, route of HIV infection, CD4 count before ART and WHO clinical stage before ART, differences in gastrointestinal reactions were statistically significant between those who initiated an ART regimen that was D4T-based, AZT-based and TDF-based compared with those whose initial ART regimen was LPV/r-based $(\mathrm{p}<0.005)$.

Among all patients in the first 3 months, 2673 of 24517 (10.9\%) patients reported having missed doses (table 5). Among those who initiated an LPV/r-based, D4T-based, AZT-based or TDF-based regimen, 13.4\%, 11.2\%, 11.4\% and $8.1 \%$ of patients respectively, reported adherence. There were significant differences between the study groups.

\section{DISCUSSION}

In this 3-year observational cohort study among HIV patients in Guangxi, China, the total mortality rate was 2.62 per 100 person-years and the total dropout rate was 4.98 per 100 person-years. The mortality rate was higher than that in developed countries and lower than previously reported rates in resource-limited settings. ${ }^{9-11}$ The total dropout rate was higher than an international, multicentre observational study in Europe, Israel and Argentina, ${ }^{12}$ but was lower than that of a Kenyan cohort study. ${ }^{11}$

In our study, initial ART regimens that were AZT-based or TDF-based were significantly superior to those that were D4T-based. Beginning in 2008, D4T was gradually replaced by AZT or TDF in China. A prospective cohort study in South Africa found that initial ART including TDF performed better than D4T overall. ${ }^{13} \mathrm{~A}$ 3-year randomised trial in South Africa, Europe and the USA showed that a regimen of TDF, 3TC and EFV was highly effective and had less toxicity than a regimen that included D4T, 3TC and EFV over 144 weeks. ${ }^{14}$ In 2010 , the WHO recommended health providers to reduce or abandon D4T, ${ }^{15} 16$ and in 2013 indicated that $\mathrm{D} 4 \mathrm{~T}$ should definitely be discontinued for use in first-line regimens due to its well-recognised metabolic toxicities. ${ }^{17}$

Previous studies have shown that LPV/r-based regimens had better virological efficacy or immunological outcome. ${ }^{18-21}$ Additionally, some studies comparing protease inhibitors (PIs) demonstrated that a combination regimen including $\mathrm{LPV} / \mathrm{r}$ was well tolerated and superior to regimens containing nelfinavir for the initial ART of adults infected with HIV. ${ }^{22}{ }^{23}$ However, our study showed that initial ART regimens that were LPV/r-based were inferior to AZT-based and TDF-based regimens. Gastrointestinal reactions and self-reported missed dose in the past 7 days were both highest among patients in our study who initiated ART with LPV/r. Gastrointestinal reactions can induce discomfort and lead to missed doses or complete discontinuation of ART. Other studies have shown similar results to our findings. For example, the EuroSIDA study 
Table 4 Viral load at 12 months of ART among HIV patients who initiated ART between 2011 and 2013 in Guangxi, China, by initial ART regimen

\begin{tabular}{|c|c|c|c|c|c|c|c|}
\hline & & VL (copies $/ \mathrm{mL})^{*}$ & & & & & \\
\hline Variable & No & $<50$ & $\%$ & OR & $P$ values & AOR $^{\star}$ & P values* \\
\hline Total & 21463 & 16139 & 75.0 & & & & \\
\hline LPV/r-based regimen & 2220 & 1633 & 73.7 & Reference & & Reference & \\
\hline D4T-based regimen & 4393 & 3180 & 72.5 & 0.94 (0.84 to 1.06$)$ & 0.29 & 0.94 (0.83 to 1.06$)$ & 0.32 \\
\hline
\end{tabular}

*Adjusted for multivariate logistic regression: age, sex, marital status, route of HIV infection, CD4 count before ART, WHO clinic stage before ART.

ART, antiretroviral therapy; AZT, zidovudine; D4T, stavudine; LPV/r, lopinavir-ritonavir; TDF, tenofovir.

found that, due to toxicity or patient choice, patients on $\mathrm{LPV} / \mathrm{r}$ had a significantly higher discontinuation rate compared with patients on NVP. ${ }^{24}$ Another study demonstrated that at week 96, the proportion of patients with virological failure who were in receiving a regimen of $\mathrm{LPV} / \mathrm{r}$ plus two nucleoside reverse-transcriptase inhibitors (NRTIs) was higher than those receiving EFV plus two NRTIs. ${ }^{25}$ In the French Hospital Database on HIV, Agence Nationale de Recherches sur le Sida, cohort 4 (FHDH-ANRS CO4) cohort study, TDF/emtricitabine (FTC) plus LPV/r was less durable than TDF/FTC with a third drug; furthermore, TDF/FTC plus LPV/r had a higher risk of non-AIDS morbidity. ${ }^{26}$ In the ART Cohort Collaboration study (ART-CC), the odds of virological failure (HIV-1 RNA level $>200$ copies/mL) at 48 weeks were higher for LPV/r compared with EFV in ART-CC. ${ }^{27}$

There are several limitations of our study. First, our study included only subjects who initiated ART, but subjects who were infected with HIV but not receiving ART were not included. Second, in this study, we used all-cause mortality and did not separate AIDS-defining death and non-AIDS-defining death, which may have an effect on the evaluation of treatment effects. Third, this study was conducted only in Guangxi, and thus might not be representative of other regions in China. Fourth, only patients who received China's free ART regimen were included in the study, and integrase inhibitors are not free in China. Thus, we could not estimate the treatment effects of integrase inhibitors.

In summary, among the patients included in Guangxi, initial ART regimens that included AZT or TDF were found to have better treatment effects than initial ART that included D4T or LPV/r. Patients that initiated an ART regiment that included $\mathrm{LPV} / \mathrm{r}$ had higher rates of gastrointestinal reaction and self-reported missed doses in the past 7 days. Thus, it is important to improve the current training for HIV care among treatment staff and enhance patient education regarding ART adherence. Future research is needed to assess the treatment effects after such improvements have been implemented.

Table 5 Adverse event, gastrointestinal reaction and adherence during the first 3 months of ART among HIV patients who initiated ART between 2011 and 2013 in Guangxi, China, by initial ART regimen

\begin{tabular}{|c|c|c|c|c|c|c|c|c|c|c|}
\hline Variable & No & $\begin{array}{l}\text { Adverse } \\
\text { event }\end{array}$ & $\%$ & P values* & $\begin{array}{l}\text { Gastrointestinal } \\
\text { reaction }\end{array}$ & $\%$ & P values* & Adherence & $\%$ & P values* \\
\hline Total & 24517 & 6966 & 28.4 & & 4203 & 17.1 & & 2673 & 10.9 & \\
\hline $\begin{array}{l}\mathrm{LPV} / \mathrm{r} \text {-based } \\
\text { regimen }\end{array}$ & 2672 & 737 & 27.6 & Reference & 613 & 22.9 & Reference & 359 & 13.4 & Reference \\
\hline $\begin{array}{l}\text { AZT-based } \\
\text { regimen }\end{array}$ & 11587 & 3666 & 31.6 & $<0.001$ & 2231 & 19.3 & 0.004 & 1324 & 11.4 & 0.16 \\
\hline $\begin{array}{l}\text { TDF-based } \\
\text { regimen }\end{array}$ & 5125 & 1163 & 22.7 & $<0.001$ & 585 & 11.4 & $<0.001$ & 416 & 8.1 & $<0.001$ \\
\hline
\end{tabular}

${ }^{\star}$ Adjusted for multivariate logistic regression: age, sex, marital status, route of HIV infection, CD4 count before ART, WHO clinic stage before ART.

ART, antiretroviral therapy; AZT, zidovudine; D4T, stavudine; LPV/r, lopinavir-ritonavir; TDF, tenofovir. 


\section{Supplementary materials}

This study also showed the number of patients lost to death, dropout, and death and dropout at the first, second, third, fourth and fifth year of ART initiation (online supplementary table 1-3). The effect of different initial ART regimens on death and dropout is shown in online supplementary table 4. Additionally, the effect of initial ART which included NNRTI-based regimen (D4T-based regimen, AZT-based regimen and TDF-based regimen) and PI-based regimen (LPV/r+3TC+D4T/ AZT/TDF) is shown in online supplementary table $5-7$.

\section{Author affiliations}

${ }^{1}$ State Key Laboratory of Infectious Disease Prevention and Control, National Center for AIDS/STD Control and Prevention (NCAIDS) , Chinese Center for Disease Control and Prevention (China CDC) , Collaborative Innovation Center for Diagnosis and Treatment of Infectious Diseases, Beijing, China

${ }^{2}$ Guangxi Center for Disease Control and Prevention, Nanning, China

Acknowledgements Data in this manuscript were collected by the Guangxi Center for Disease Control and Prevention. Principal investigators for this study included ZT, ZS and QZ (Guangxi Center for Disease Control and Prevention).

Contributors RK, LL, HC, YR, WY were responsible for study design and planning. RK, LL, HC, QZ, JZ, ZS, GL, ZT, YS, YR, WY contributed to data collection and analysis. RK, LL, HX, YS, YR, WY contributed to interpretation of data. RK, YS, YR, WY contributed to writing the manuscript. All authors read and approved the final version of the manuscript.

Funding This study was supported by the Guangxi Medical and Health Project (Z20170126), Guangxi Science and Technology Bureau (Grant AB16380213), National Natural Science Foundation of China (Grants 81502862, 81460510 and 81360442), Guangxi Bagui Honor Scholarship, Ministry of Science and Technology of China (2017ZX10201101, 2018ZX10721102-006) and Chinese State Key Laboratory of Infectious Disease Prevention and Control.

Competing interests None declared.

Patient consent for publication Obtained.

Ethics approval The institutional review board (IRB) of the NCAIDS, China CDC approved this study.

Provenance and peer review Not commissioned; externally peer reviewed. Data sharing statement No additional data are available.

Open access This is an open access article distributed in accordance with the Creative Commons Attribution Non Commercial (CC BY-NC 4.0) license, which permits others to distribute, remix, adapt, build upon this work non-commercially, and license their derivative works on different terms, provided the original work is properly cited, appropriate credit is given, any changes made indicated, and the use is non-commercial. See: http://creativecommons.org/licenses/by-nc/4.0/.

\section{REFERENCES}

1. Zhang $F$, Haberer JE, Wang $Y$, et al. The Chinese free antiretroviral treatment program: challenges and responses. AIDS 2007;21(Suppl 8):S143-S148.

2. Zhang FJ, Pan J, Yu L, et al. Current progress of China's free ART program. Cell Res 2005;15:877-82.

3. National center for AIDS/STD Control and Prevention, China CDC. Manual of the national free antiretroviral treatment. 1st edn, 2005. http://www.ncaids.chinacdc.cn/ls/m/200501/t20050131_1075189. html

4. Book writing group. Manual of the national free antiretroviral treatment. 2nd edn. Beijing: People's Medical Publishing House, 2008.
5. National center for AIDS/STD Control and Prevention, China CDC. Manual of the national free antiretroviral treatment. 4th edn. Beijing: People's Medical Publishing House, 2016.

6. UNAIDS. An ambitious treatment target to help end the AIDS epidemic: UNAIDS, 2014. http://www.aidsdatahub.org/ambitioustreatment-target-help-end-aids-epidemic-unaids-2014

7. National center for AIDS/STD Control and Prevention, China CDC. Annual Report on Provincial AIDSSTD Surveillance in 2017.

8. Ma Y, Zhang F, Zhao Y, et al. Cohort profile: the Chinese national free antiretroviral treatment cohort. Int J Epidemiol 2010;39:973-9.

9. Braitstein P, Brinkhof MW, Dabis F, et al. Mortality of HIV-1-infected patients in the first year of antiretroviral therapy: comparison between low-income and high-income countries. Lancet 2006;367:817-24.

10. Bygrave $\mathrm{H}$, Ford $\mathrm{N}$, van Cutsem $\mathrm{G}$, et al. Implementing a tenofovirbased first-line regimen in rural Lesotho: clinical outcomes and toxicities after two years. J Acquir Immune Defic Syndr 2011;56:e75-e78.

11. Karcher $\mathrm{H}$, Omondi A, Odera J, et al. Risk factors for treatment denial and loss to follow-up in an antiretroviral treatment cohort in Kenya. Trop Med Int Health 2007;12:687-94.

12. Mocroft A, Kirk O, Aldins P, et al. Loss to follow-up in an international, multicentre observational study. HIV Med 2008;9:261-9.

13. Velen K, Lewis JJ, Charalambous S, et al. Comparison of tenofovir, zidovudine, or stavudine as part of first-line antiretroviral therapy in a resource-limited-setting: a cohort study. PLoS One 2013;8:e64459.

14. Gallant JE, Staszewski S, Pozniak AL, et al. Efficacy and safety of tenofovir DF vs stavudine in combination therapy in antiretroviralnaive patients: a 3-year randomized trial. JAMA 2004;292:191-201.

15. World Health Organization. National anti-retroviral therapy guideline. 2009 http://www.who.int/hiv/pub/guidelines/nepal_art.pdf?ua=1

16. World Health Organization. Rapid advice antiretroviral therapy for HIV infection in adults and adolescents. 2009 http://www.who.int/hiv/ pub/arv/advice/en

17. World Health Organization. Consolidated guidelines on the use of antiretroviral drugs for treating and preventing HIV infection recommendations for a public health approach. $2013 \mathrm{http} / / / a p p s$. who.int/iris/handle/10665/85321

18. Huang $\mathrm{X}, \mathrm{Xu} \mathrm{Y}$, Yang $\mathrm{Q}$, et al. Efficacy and biological safety of lopinavir/ritonavir based anti-retroviral therapy in HIV-1-infected patients: a meta-analysis of randomized controlled trials. Sci Rep 2015;5:8528.

19. Wolf E, Trein A, Baumgarten A, et al. 144-week outcomes of lopinavir/ritonavir (LPV/r)-based first-line ART in 1,409 HIV-infected patients: data from the German STAR/STELLA cohort. J Int AIDS Soc 2014;17(4 Suppl 3):19770.

20. Torti C, Maggiolo F, Patroni A, et al. Exploratory analysis for the evaluation of lopinavir/ritonavir-versus efavirenz-based HAART regimens in antiretroviral-naive HIV-positive patients: results from the Italian MASTER Cohort. J Antimicrob Chemother 2005;56:190-5.

21. Potard V, Rey D, Mokhtari S, et al. First-line highly active antiretroviral regimens in 2001-2002 in the French Hospital Database on HIV: combination prescribed and biological outcomes. Antivir Ther 2007;12:317-24.

22. Kempf DJ, King MS, Bernstein B, et al. Incidence of resistance in a double-blind study comparing lopinavir/ritonavir plus stavudine and lamivudine to nelfinavir plus stavudine and lamivudine. $J$ Infect Dis 2004;189:51-60.

23. Walmsley S, Bernstein B, King M, et al. Lopinavir-ritonavir versus nelfinavir for the initial treatment of HIV infection. N Engl J Med 2002;346:2039-46.

24. Reekie J, Reiss P, Ledergerber B, et al. A comparison of the longterm durability of nevirapine, efavirenz and lopinavir in routine clinical practice in Europe: a EuroSIDA study. HIV Med 2011;12:259-68.

25. Riddler SA, Haubrich R, DiRienzo AG, et al. Class-sparing regimens for initial treatment of HIV-1 infection. $N$ Engl J Med 2008;358:2095-106.

26. Potard V, Rey D, Poizot-Martin I, et al. Lopinavir/r no longer recommended as a first-line regimen: a comparative effectiveness analysis. J Int AIDS Soc 2014;17:19070.

27. Mugavero MJ, May M, Ribaudo HJ, et al. Comparative effectiveness of initial antiretroviral therapy regimens: ACTG 5095 and 5142 clinical trials relative to ART-CC cohort study. J Acquir Immune Defic Syndr 2011;58:253-60. 